\title{
Leiomyosarcoma of the inferior vena cava: Survival rate following radical resection
}

\author{
FREDERICO JOSÉ RIBEIRO TEIXEIRA $\mathrm{Jr}^{1,2}$, SERGIO DIAS DO COUTO NETTO ${ }^{2}$, \\ ANDRÉ LUIS DE FREITAS PERINA ${ }^{1}$, FABIO C. M. TORRICELLI ${ }^{3}$, LUCIANA RAGAZZO TEIXEIRA ${ }^{4}$, \\ ANTONIO EDUARDO ZERATI ${ }^{4}$, FABIO DE OLIVEIRA FERREIRA ${ }^{1}$, EDUARDO HIROSHI AKAISHI ${ }^{1}$, \\ WILLIAM CARLOS NAHAS ${ }^{3}$ and EDIVALDO MASSAZO UTIYAMA ${ }^{2}$
}

${ }^{1}$ Sarcoma and Melanoma Surgery Unit, São Paulo Cancer Institute; Departments of ${ }^{2}$ General Surgery, ${ }^{3}$ Urology and
${ }^{4}$ Vascular Surgery, Clinics Hospital, University of São Paulo Medical School, Cerqueira César, SP 05403-000, Brazil

Received October 15, 2016; Accepted March 7, 2017

DOI: $10.3892 / \mathrm{ol} .2017 .6706$

\begin{abstract}
Leiomyosarcoma (LMS) of inferior vena cava (IVC) is a rare neoplasm affecting approximately $1 / 100,000$ people. The prognosis is poor and potential curative intent occurs through challenging operations, such as vena cava resection, occasionally multivisceral when required, and vascular reconstruction. There are few retrospective series regarding this retroperitoneal neoplasm, and the aim of the present study was to discuss the experience at the São Paulo Cancer Institute and Clinics Hospital of University of São Paulo Medical School, São Paulo, Brazil. The current study is a retrospective review of 7 patients treated in the two tertiary hospitals between 2005 and 2013. Oncological and operative aspects were discussed, primarily regarding surgical aspects highlighting en bloc resection, vascular reconstruction, and the overall survival and recurrence rates. All the patients were treated with radical intent, 4 of whom underwent multivisceral resection, with the kidney being the most resected organ. The location of the IVC tumor was described using Kulaylat's description and the median tumor size was $10 \mathrm{~cm}$. Vascular reconstruction was necessary in 4 patients. The overall survival rate at 3 and 5 years was 100 , and $25 \%$, respectively. The disease-free survival rate at 3 and 5 years was 57 and 20\%, respectively. In conclusion, IVC LMS is a rare and severe retroperitoneal neoplasm, with multivisceral resections remaining a surgical challenge. The treatment requires numerous experienced surgeons and the impact of microscopic free margins remains
\end{abstract}

Correspondence to: Dr Sergio Dias do Couto Netto, Department of General Surgery, Clinics Hospital, University of São Paulo Medical School, 255 Enéas Carvalho de Aguiar Avenue, Cerqueira César, SP 05403-000, Brazil

E-mail: sergiodcnetto@gmail.com

Key words: leiomyosarcoma, inferior vena cava sarcoma, retroperitoneal sarcoma, retroperitoneal tumor, sarcoma, vena cava tumor unclear. Vascular reconstruction depends on several aspects regarding primarily the topography of the tumor.

\section{Introduction}

Leiomyosarcoma (LMS) of the inferior vena cava (IVC) is a rare type of neoplasm, accounting for $\sim 0.5 \%$ of adult soft tissue sarcoma, affecting $<1 / 100,000$ of all adult malignancies (1-3). The prognosis is poor, as patients present with intra or extra-luminal growth often with invasion of adjacent structures. According to a recent pooled data analysis, $<400$ cases of IVC LMS have been reported, with the majority of studies limited to single case reports or compilations of a case series (4). The 5-year survival rate ranges between 31 and $66.7 \%$ for patients with IVC LMS following complete macroscopic resection (1,5-9).

LMS of the IVC are usually presented as large tumors at the time of diagnosis. In several studies, tumors were $>10 \mathrm{~cm}(1-3,8,10)$. Occasionally, LMS of the IVC occurs in young patients, with few or no comorbidities, as localized disease. They are predominant in females aged 54 years at the time of diagnosis (11), IVC reconstruction may be considered once long-term survival can be accomplished in patients submitted to $\mathrm{R} 0$ resection $(3,9)$.

Surgery is currently the only potentially curative therapy. The paramount aim when approaching IVC LMS include achieving local control, maintaining the patency of major venous flow and identifying the most effective adjuvant therapeutic strategies to reduce the recurrence rate. However, the technical challenges presented by anatomical characteristics of this disease raise important issues, such as the role of multivisceral resection and vascular options of reconstructions. The clinical expertise on radical resection and venous reconstruction remains limited and data regarding multimodal therapies, such as chemotherapy (CT), radiation therapy (RT), or both (CRT) in combination with surgical resection are scarce, with the optimal treatment strategy remaining unclear.

In the present study, a series of seven patients submitted to operative treatment of primary LMS of the IVC was reviewed, and the effect of multivisceral resection on survival rate and the options of venous reconstruction were analyzed. 


\section{Materials and methods}

A retrospective review was performed on the medical records of all the patients treated with upfront resection of primary IVC LMS over a five-year period between June/2007 and October/2013. Variables collected from the medical records included demographic and clinical data, tumor location along the IVC, adjuvant therapies received, surgical technique employed, and the surgical pathology report. Segments of IVC affected by the tumor were classified according to Kulaylat et al (12) as shown in Fig. 1, and tumor histology grade (I, II, III) was reported according to the FNCLCC (Fédération Nationale des Centres de Lutte Contre le Cancer or French Federation of Cancer Centers Sarcoma Group) (13). Multi-visceral resection was defined according to the en bloc model $(14,15)$ of tumor resection with adjacent tissue graft reconstruction. Furthermore, data on the 30-day mortality, 30-day complication, disease-free survival (DFS) and overall survival (OS) rates, and the site of recurrence were collected.

The primary endpoints of the study were postoperative mortality and morbidity, and OS rate. Other variables, such as status of resection secondary to radical resection (R0, R1 or $\mathrm{R} 2$ resections) and the patency of the graft utilized in the vascular reconstruction were reported as secondary endpoints.

The classification by Kulaylat et al (12) for an IVC tumor was classified according to the level in the IVC: segment I, infrarenal; segment II, inter- and supra-renal up to but not including the main suprahepatic veins; and segment III, suprahepatic with possible intracardiac extension.

OS rates were calculated using the time between the date of surgery and the date of mortality or last contact. DFS was defined as the time to local or distant tumor recurrence following initial treatment. Kaplan-Meier estimator survival curves for OS and DFS were determined (16). The patients were followed up until they revealed the outcome of interest or censoring by the date of the last follow-up.

\section{Results}

Patient 1. A 78-year-old male with a 4-month history of abdominal pain was admitted to the pancreas and biliary tract surgery service at the Clinics Hospital affiliated to the University of São Paulo (São Paulo, Brazil). A computed tomography (CT) scan (Fig. 2) of the abdomen revealed a $7.0 \mathrm{~cm}$ mass between the IVC and duodenum, which was suspected as a primary IVC tumor. The patient was referred to the surgical oncology group at the São Paulo Cancer Institute (São Paulo, Brazil) for further evaluation. The vascular surgery and surgical oncology teams planned the operative resection together. Exploration was performed via a midline incision. The mass was identified arising from the IVC and involved the lower and middle segment, and the right renal vein. Proximal and distal controls from the IVC were obtained (Fig. 3), and the mass and the right kidney were dissected free. The tumor was excised en bloc with $9.5 \mathrm{~cm}$ of the IVC and the right kidney with a $10 \mathrm{~mm}$ margin. IVC reconstruction was performed using an 18-mm Dacron prosthetic graft. Left renal vein reconstruction using a polytetrafluoroethylene (PTFE) prosthetic graft was performed (Fig. 4). The patient was heparinized. While on systemic anticoagulation, the patient was submitted to reoperation due to a postoperative retroperitoneal hematoma. The hematoma was evacuated and no active bleeding was identified. The patient was discharged home on warfarin. Surgical pathological examination demonstrated a multilobulated high-grade $8.0 \mathrm{~cm}$ LMS of the IVC with negative surgical margins. The patient remained well for 28 months; however, multiple pulmonary nodules were identified in the routine surveillance CT scan. The patient subsequently underwent multiple metastasectomies with subsequent recurrences. The patient succumbed to the disease 57 months following the original resection.

Patient 2. A 68-year-old female with a six-month history of right upper quadrant pain was found to have a $20 \mathrm{~cm}$ sub-hepatic mass at the level of the right renal hilum. The patient was admitted to the Heart Institute at the University of São Paulo and was referred to the general surgical oncology group for further evaluation. A CT scan of the abdomen revealed the mass arising from the vena cava and extending into the lower segment of the IVC, with compression of the right renal vein. Operative resection was similar to the first case. Exploration was performed via a Mercedes incision, and the tumor was identified along the IVC just below the liver and the right renal vein. Proximal and distal controls from the cava were obtained, and the mass and the right kidney were dissected free. The IVC was clamped inferiorly $4 \mathrm{~cm}$ above the bifurcation of the common iliac veins and superiorly in the infra-hepatic segment of the IVC. The left renal vein was sectioned near the IVC. A large mass was removed with $20 \mathrm{~cm}$ of the IVC en bloc with the right kidney. Reconstruction of the IVC was performed using a $20-\mathrm{mm}$ Dacron graft and reconstruction of the left renal vein was performed with an end-to-side anastomosis using a 6-mm PTFE graft. The postoperative course of the patient was unremarkable. Surgical pathological examination demonstrated a grade I (FNCLCC) 18x15x13 cm LMS of the IVC with negative surgical margins. The patient was discharged on therapeutic enoxaparin. There was no evidence of disease at the time of this report 39 months following the initial surgery.

Patient 3. A 34-year-old female with a six-month history of right upper quadrant pain was found to have an $11 \mathrm{~cm}$ sub-hepatic mass, which was suspected as a primary IVC tumor. A CT scan of the abdomen revealed a mass arising from the vena cava and extending into the lower segment of the IVC. Intraluminal filling defects were detected, indicating the invasion of the tumor into the IVC and bilateral renal veins (Fig. 5). Vascular surgery and surgical oncology groups planned the operative resection together. Following confirmation of the clinical staging of the localized disease, the patient underwent surgery and a bilateral subcostal incision combined with a median extension was performed. The tumor was dissected free intra-abdominally and the liver was mobilized. Thus, the IVC and bilateral renal veins were clamped and the tumor was subsequently removed with a partial resection of the subsegment IVb of the liver. The distal end of the IVC was ligated and excluded. The proximal segment of the IVC was anastomosed to an 18-mm Dacron graft with 8-mm bilateral arms, 


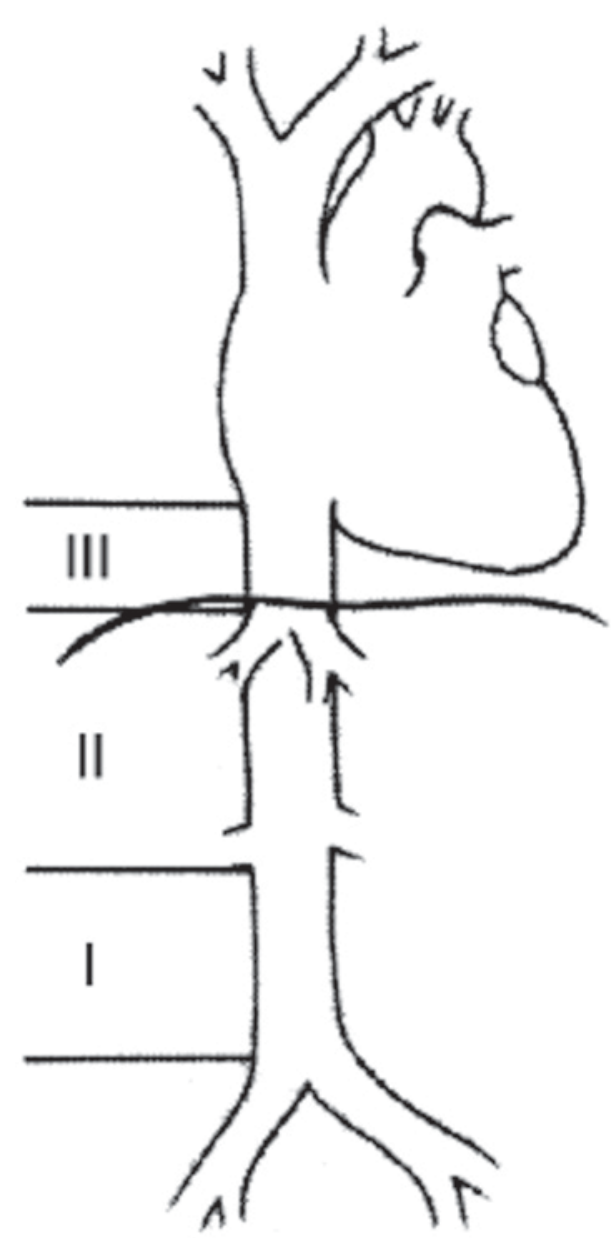

Figure 1. The classification of vena cava sarcoma according to Kulaylat et al (12).

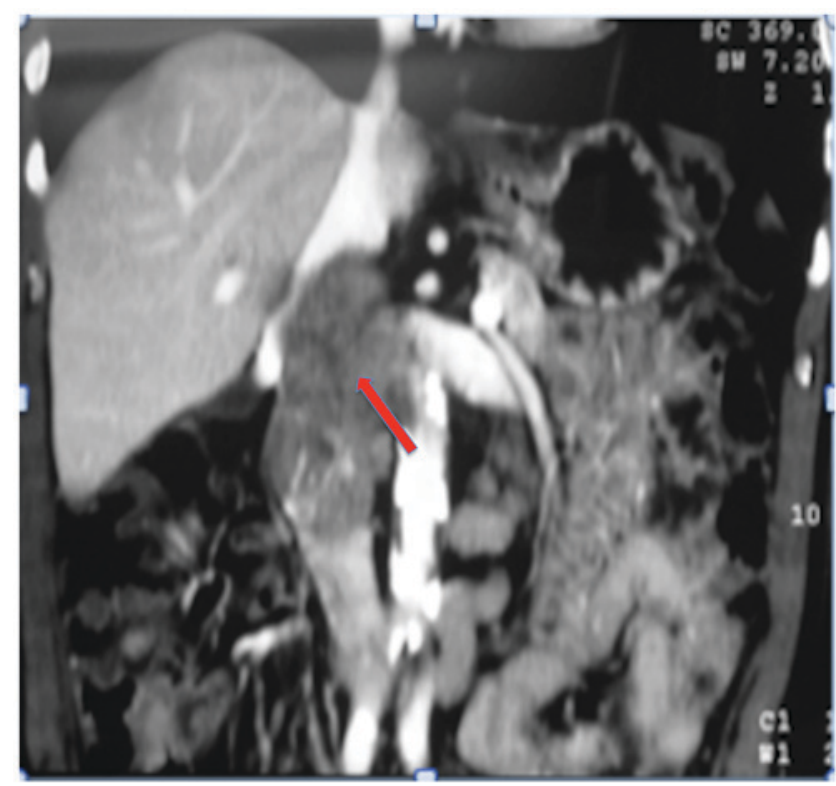

Figure 2. Abdominal computed tomography scan revealing an inferior vena cava sarcoma infiltrating the right renal vessels, indicated by the arrow.

which were anastomosed to the bilateral renal veins (Fig. 6). Surgical pathological examination demonstrated a grade III (FNCLCC) $12 \mathrm{~cm}$ LMS of the IVC with negative surgical

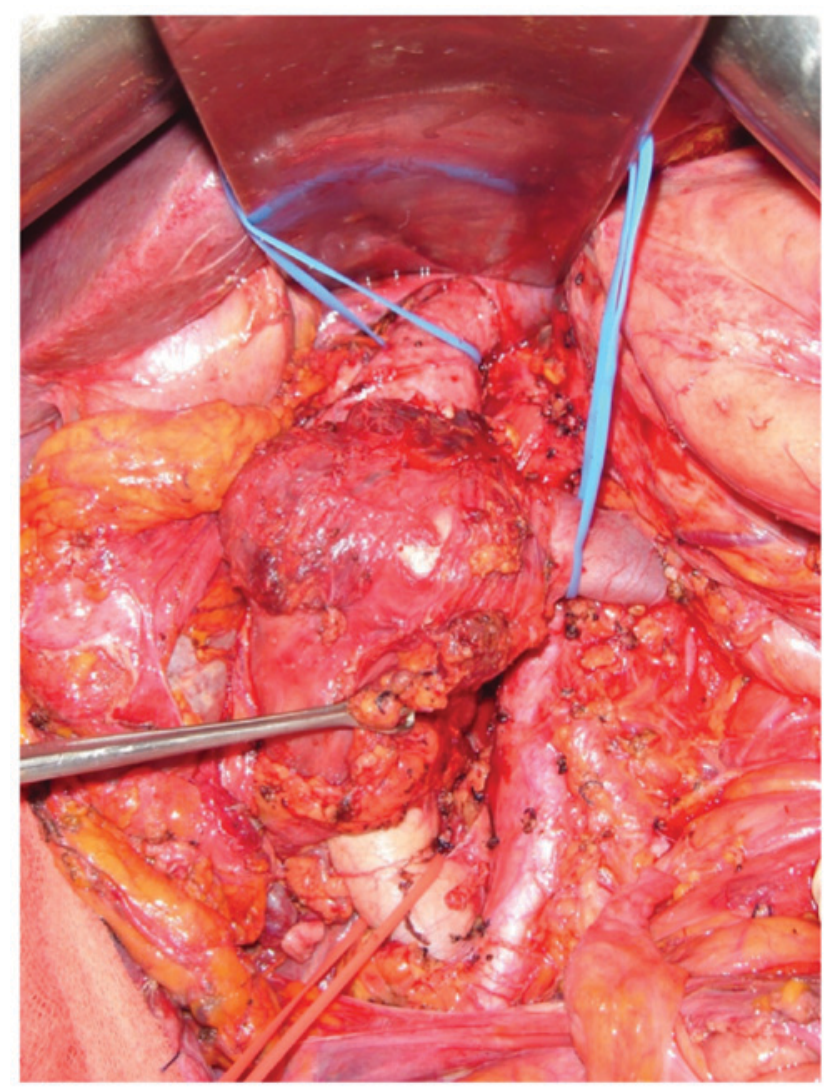

Figure 3. Retroperitoneal tumor dissected in a patient with leiomyosarcoma of the inferior vena cava.

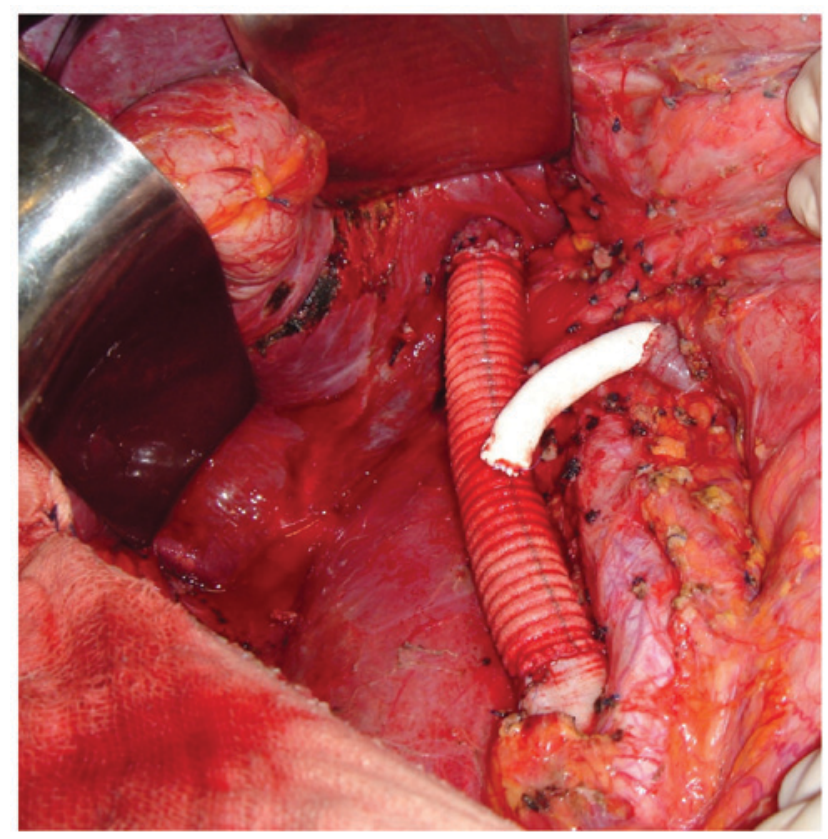

Figure 4. Image captured during inferior vena cava reconstruction of a patient with leiomyosarcoma.

margins. The patient had an uneventful postoperative recovery and was discharged home on the 22nd postoperative day in good condition. Oral warfarin therapy was administered for 6 months following discharge. After a follow-up thoracic and abdominal CT scan 14 months postoperatively, the patient was 


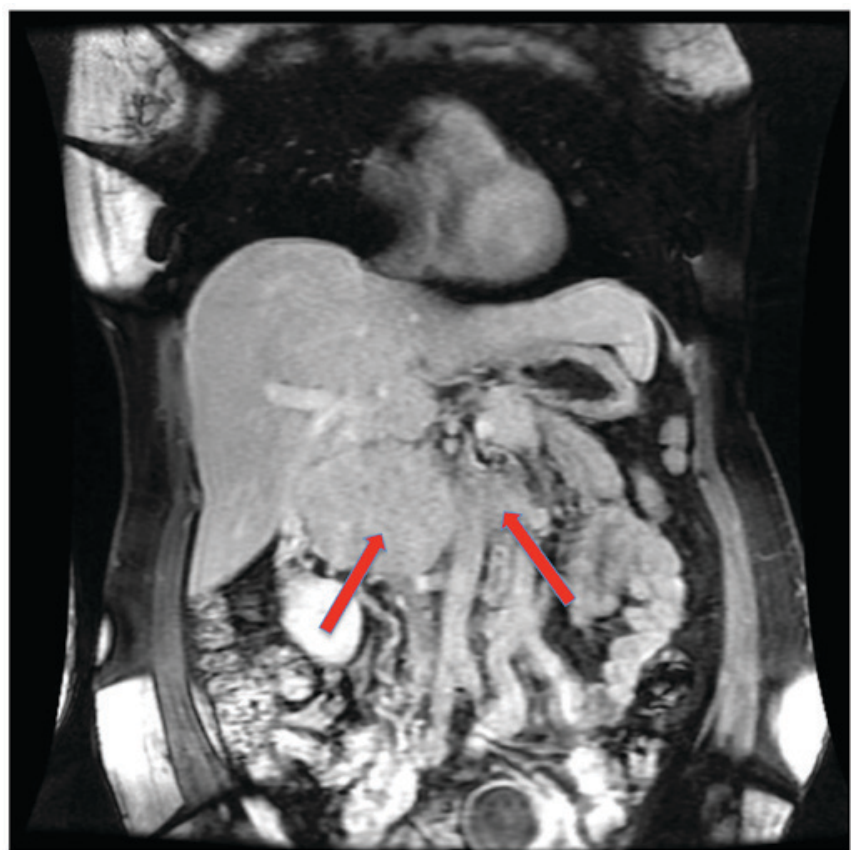

Figure 5. Computed tomography scan revealing an inferior vena cava sarcoma infiltrating both renal vessels. The arrows highlight the vessel's invasion.

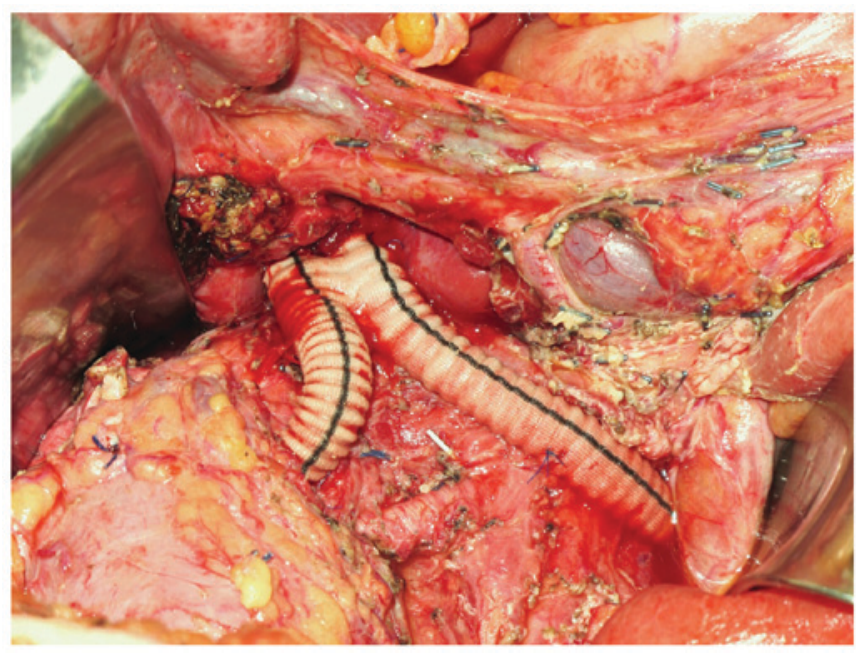

Figure 6. Image captured during inferior vena cava reconstruction of a patient with leiomyosarcoma.

diagnosed with multiple pulmonary and hepatic metastases. Systemic treatment with chemotherapy began and the patient is alive with disease as of the last visit.

Patient 4. An 81-year-old female patient, who had atrial fibrillation and was anticoagulated, was present with vague abdominal pain for 3 months. The CT scan demonstrated an $\sim 5 \mathrm{~cm}$ mass arising from the IVC and inferior to the right renal vein with no other associated abnormalities. The patient underwent surgical resection of the mass, including complete resection of the IVC below the level of the renal veins. No reconstruction was performed. The patient had an uneventful postoperative recovery. Pathological analysis revealed a high-grade $5.3 \mathrm{~cm}$ LMS of the IVC with negative surgical margins. The patient has no evidence of disease at the time of this report, 69 months following the initial surgery. However, at 42 months the patient appeared in the emergency department with vaginal bleeding. The pelvic CT scan revealed an exuberant collateral circulation and multiple pelvic varices. The bleeding stopped spontaneously. At present, the patient is doing well with no more episodes of bleeding.

Patient 5. A 53-year-old female patient presented with right lumbar pain for 2 months. An abdominal ultrasonography revealed a $4.5 \times 4.5 \mathrm{~cm}$ mass, which was suspected as a primary tumor of the right kidney. The patient was admitted to the urology service at the Clinics Hospital. The CT scan demonstrated a $6.9 \times 6.3 \mathrm{~cm}$ tumor between the pancreas head, right kidney and liver, which was most probably a primary IVC tumor. Preoperative endoscopic eco-guided biopsy revealed fusiform cells with no atypia. The urology and vascular surgery teams planned the operative procedure together. Exploration was performed via a right subcostal incision. Complete resection of the IVC below the level of the renal veins was performed. Surgical margins were negative. IVC reconstruction was performed using a 20-mm Dacron prosthetic graft. Pathological analysis revealed an $8.0 \mathrm{~cm}$ grade II (FNCLCC) LMS of the IVC. The postoperative course was unremarkable. Therapeutic anticoagulation with enoxaparin was performed within 6 months. Thrombosis of the graft was identified in an abdominal CT 3 months following resection. A thoracic CT scan 4 months following surgery demonstrated multiple small pulmonary nodules, which was suspected as pulmonary metastasis. The last follow-up was 38 months following diagnosis. The patient succumbed to the disease.

Patient 6. A 49-year-old female patient presented with right lumbar pain for 1 month. The CT scan demonstrated a $9.2 \mathrm{~cm}$ retroperitoneal mass suspected to be a right primary adrenal tumor with invasion of the IVC. Biopsy guided by imaging revealed an LMS. Surgical exploration was performed via a bilateral subcostal incision. IVC ligation below the tumor was performed, as well as ligation of the left renal vein. The retrohepatic IVC was sutured with vascular reconstruction. The tumor was removed en bloc with the right kidney and adrenal gland. Pathological analysis revealed a $10.0 \times 9.9 \times 6.5 \mathrm{~cm}$ grade III (FNCLCC) LMS of the IVC invading the renal hilum and parenchyma, and the adrenal gland. One microscopic positive surgical margin was identified. Progression to pulmonary metastasis was detected 8 months postoperatively. The patient succumbed to the disease 48 months following resection of the primary tumor.

Patient 7. A 53-year-old female patient presented with abdominal pain. The CT scan revealed a heterogeneous $10.0 \times 9.0 \times 7.0 \mathrm{~cm}$ mass adjacent to the right kidney and invading the posterior wall of the IVC. Following clinical staging, a complete macroscopic resection was performed by the surgical urology team with ligation of the IVC just below the right renal vein. No vascular reconstruction was attempted. Pathological analysis revealed an $11.5 \times 7.2 \times 6.5$ grade II (FNCLCC) LMS of the IVC with narrow margins. The CT scan 4 months postoperatively revealed 

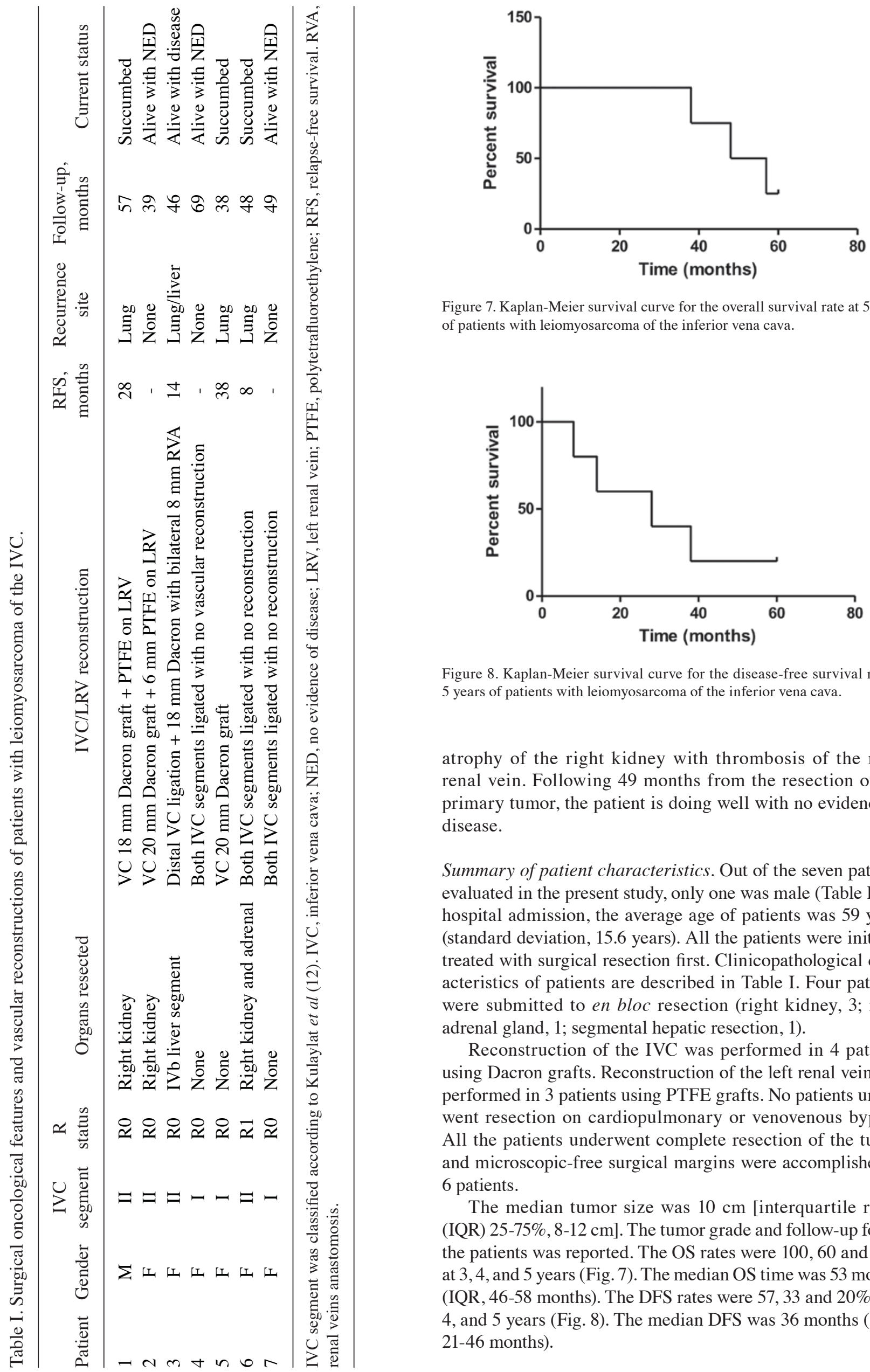

Figure 7. Kaplan-Meier survival curve for the overall survival rate at 5 years of patients with leiomyosarcoma of the inferior vena cava.

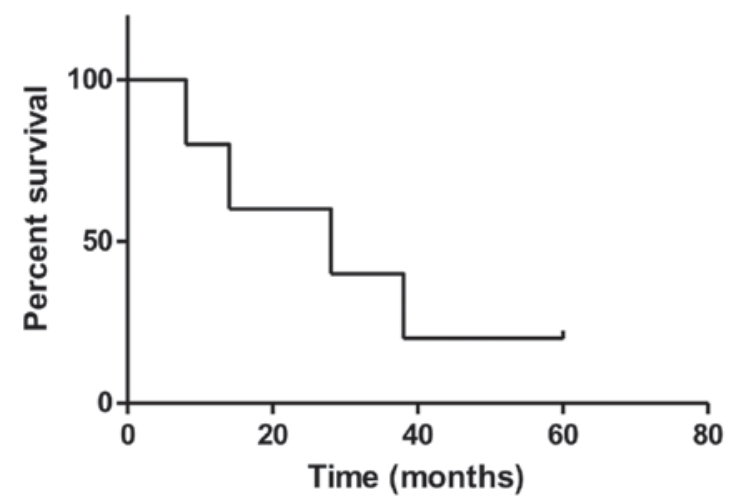

Figure 8. Kaplan-Meier survival curve for the disease-free survival rate at 5 years of patients with leiomyosarcoma of the inferior vena cava.

atrophy of the right kidney with thrombosis of the right renal vein. Following 49 months from the resection of the primary tumor, the patient is doing well with no evidence of disease.

Summary of patient characteristics. Out of the seven patients evaluated in the present study, only one was male (Table I). At hospital admission, the average age of patients was 59 years (standard deviation, 15.6 years). All the patients were initially treated with surgical resection first. Clinicopathological characteristics of patients are described in Table I. Four patients were submitted to en bloc resection (right kidney, 3; right adrenal gland, 1; segmental hepatic resection, 1).

Reconstruction of the IVC was performed in 4 patients using Dacron grafts. Reconstruction of the left renal vein was performed in 3 patients using PTFE grafts. No patients underwent resection on cardiopulmonary or venovenous bypass. All the patients underwent complete resection of the tumor and microscopic-free surgical margins were accomplished in 6 patients.

The median tumor size was $10 \mathrm{~cm}$ [interquartile range (IQR) $25-75 \%, 8-12 \mathrm{~cm}$ ]. The tumor grade and follow-up for all the patients was reported. The OS rates were 100, 60 and 25\% at 3,4 , and 5 years (Fig. 7). The median OS time was 53 months (IQR, 46-58 months). The DFS rates were 57, 33 and $20 \%$ at 3 , 4 , and 5 years (Fig. 8). The median DFS was 36 months (IQR, 21-46 months). 


\section{Discussion}

LMS is one of the most frequent types of retroperitoneal soft tissue sarcoma. For all newly diagnosed soft tissue sarcoma, the estimated incidence of LMS ranges between 10 and $20 \%$ (17). However, vascular LMS constitutes $1-2 \%$ of all soft tissue sarcoma and is associated with poor prognosis (18). In a postmortem examination in 1871, Perl et al (19) described the first LMS of the IVC. Since then, $<400$ cases have been reported in the English literature $(3,4)$. This rare type of tumor originates from the smooth muscle of the venous wall. Histological description revealed that they are composed of fascicles of spindle cells. The nuclei are hyperchromatic and there is abundant eosinophilic cytoplasm. The pleomorphism is exuberant and occasionally resembles an undifferentiated soft tissue sarcoma (20). LMS of the IVC predominates in females. Its incidence peak is in the fifth decade of life $(>75 \%$ of the cases reported). The female/male ratio is $\sim 3: 1$ (4).

Macroscopic surgical resection is the primary curative treatment for patients with localized disease. This is the only potentially curative therapy since the first resection of the LMS of the IVC at Lexington Memorial Hospital in Chicago, in 1951 (21). However, complete surgical removal of LMS of the IVC does not confer long-term survival for all patients. Furthermore, the role of the multivisceral resections remains controversial. Adjuvant therapeutic strategies, such as systemic CT, RT (preoperative, postoperative or intraoperative) or CRT are considered; however, the clinical benefits of these treatments have not been clearly determined (5). The role of vascular reconstruction of the IVC remains controversial. There is no consensus regarding which option should be elected (primary repair, ligation or reconstruction). All venous surgical treatment options have been previously utilized (1,3,7,22-24).

The majority of patients described in the present study presented with abdominal pain, which is the first indication for upfront resection when feasible. Approximately $60 \%$ of the patients presented with non-specific abdominal pain (4). Survival rates are more improved when patients are treated with extended resection compared with those who are managed with medical therapy alone $(1,3)$. For patients treated with non-surgical therapy, prognosis is poor and survival time is measured in months $(3,25)$. It appears that complete macroscopic resection confers the longest survival rates. Hollenbeck et al (1) reviewed a series of 25 patient cases that underwent excisional therapy. A 3-year OS rate of $76 \%$ was observed for patients with LMS of the IVC that had been completed removed and 0\% OS was observed when incomplete resections were performed (1). In a previous case report on 14 patients, Hines et al (23) reported a 5-year survival rate of $68 \%$ for patients with negative margins identified upon pathological analysis compared with $0 \%$ for patients with positive margins. Generally, a tumor free margin of $1 \mathrm{~cm}$ is necessary for soft tissue sarcoma. The proximity with various organs may demand an en bloc multi-organ resection involving anatomical structures, such as the aorta, kidneys, adrenal, liver and colon (26).

In the present study, $\sim 50 \%$ of patients underwent en bloc resection of the tumor with $\geq 1$ adjacent organs in order to obtain free surgical margins. Right nephrectomy $(60 \%)$, right adrenalectomy (27\%) and partial hepatectomy (20\%) are the most of the resected organs. En bloc resection was demonstrated to be associated with a decrease in OS rate in a recent pooled data analysis of 377 patients (4). The effect of clear margins on OS rate remains unclear, according to studies conducted by Wachtel et al (4) and Hines et al (23), the effect of R0 or R1 resections OS and DFS rates are indifferent. However, macroscopic positive margins have been identified to be associated with worse prognosis, $\sim 0 \%$ in 5 years, addressing the role of multivisceral resection in appropriate cases.

In the present case series, the median survival of 21 months confirmed that radical resections of the LMS of the IVC with the intention of obtaining a complete macroscopic resection with negative margins should be the aim for those with localized disease and acceptable clinical performance. All the patients achieved complete macroscopic resections and only one patient had microscopic positive surgical margins. This patient succumbed to the disease 48 months following resection of the primary tumor. The results of previous studies have revealed that microscopic positive surgical margins have no effect on DFS and OS rates $(1,8,23)$.

One issue following radical resection is the reconstruction of the IVC and occasionally the left renal vein. Several options exist when vascular reconstruction is considered (5,27-31). Small caval defects can be closed with primary sutures or with a saphenous patch. However, in numerous patients circumferential resection of the IVC is required. When a complete IVC thrombosis has been revealed and the tumor of the IVC is localized to level I, vascular reconstruction may not be necessary. Collateral circulation is usually present and when patients develop leg edema, in general, it is well tolerated. Some advantages of this approach are the reduced operative time, no risk of synthetic graft infection and no need for prolonged anticoagulation, reducing the risk of reoperation due to bleeding. One of the patients discussed in the present report developed a retroperitoneal hematoma secondary to systemic anticoagulation requiring re-laparotomy. This has also been reported in other studies (4).

In a long-term follow-up, no lower extremity edema was observed in patients who underwent IVC reconstructions in the current series. In a previous study, lower extremity edema was considered significant in $50 \%$ of patients when no IVC reconstruction is undertaken (26). Late patency of the synthetic tube graft was observed in $75 \%$ of patients and no significant lower extremity edema was observed when thrombosis of the graft occurred (29). A previous study revealed patency rates of $95 \% 5$ years following IVC reconstruction (32). In the present study, ligation of the IVC was performed in one patient and following several months the patient developed pelvic varices, and presented in the emergency department with vaginal bleeding secondary to varices. It was an isolated event without hemodynamic instability and the bleeding stopped spontaneously. Whether or not IVC reconstruction may prevent this type of complication in long-term survival patients remains unclear, but the long-term complications secondary to IVC ligation are of concern (23).

When the patients presented with a patent IVC, vascular reconstructions were performed, as identified by previous results $(8,27,29,30)$. We suggest maintaining the venous return using synthetic tube grafts whenever the patency of the IVC 
is confirmed preoperatively through diagnostic imaging tests. For patients with level II LMS of the IVC the option to perform IVC reconstruction considers vicariation of the collateral circulation to guarantee the venous return of the left kidney. The IVC and bilateral renal veins can be excluded through ligation when preoperative imaging tests revealed exuberant vicariation of collateral vessels (33). However, the risk of acute renal failure following right nephrectomy and ligation of the left renal vein is a concern. Vascular prosthesis to reconstruct the left renal vein is recommended to maintain the venous outflow and avoid renal dysfunction (34-36).

In the present study, two patients with level II LMS underwent an end-to-side anastomosis between the left renal vein and an 18-mm Dacron prosthesis was used to reconstruct the IVC. A 6-mm PTFE between the left renal vein and Dacron were chosen to accomplish the left renal outflow. In another patient with level II LMS of the IVC it was possible to remove the tumor and maintain both kidneys. The distal end of the IVC was ligated and excluded, and the bilateral veins were clamped. Vascular reconstruction was performed creating an anastomosis between the cranial stump of the IVC and an 18-mm Dacron graft was used with bilateral 8-mm arms that were anastomosed to the bilateral renal veins. This type of reconstruction has previously been reported in other studies (34). All 3 patients remained asymptomatic and did well throughout follow-up without renal dysfunction.

In conclusion, LMS of the IVC is a rare retroperitoneal sarcoma, and radical resection is the only therapeutic option capable of conferring long-term survival. To obtain complete macroscopic resection, removal of adjacent organs is usually necessary. Microscopic-free surgical margins are necessary but its effect on long-term survival remains unclear. Venous reconstruction is selectively indicated. There is no consensus, but in general, when partial obstruction of the IVC occurs the reconstruction of the IVC is encouraged.

\section{References}

1. Hollenbeck ST, Grobmyer SR, Kent KC and Brennan MF: Surgical treatment and outcomes of patients with primary inferior vena cava leiomyosarcoma. J Am Coll Surg 197: 575-579, 2003.

2. Laskin WB, Fanburg-Smith JC, Burke AP, Kraszewska E, Fetsch JF and Miettinen M: Leiomyosarcoma of the inferior vena cava: Clinicopathologic study of 40 cases. Am J Surg Pathol 34: $873-881,2010$

3. Mingoli A, Cavallaro A, Sapienza P, Di Marzo L, Feldhaus RJ and Cavallari N: International registry of inferior vena cava leiomyosarcoma: Analysis of a world series on 218 patients. Anticancer Res 16: 3201-3205, 1996.

4. Wachtel H, Gupta M, Bartlett EK, Jackson BM, Kelz RR, Karakousis GC, Fraker DL and Roses RE: Outcomes after resection of leiomyosarcomas of the inferior vena cava: A pooled data analysis of 377 cases. Surg Oncol 24: 21-27, 2015.

5. Ito H, Hornick JL, Bertagnolli MM, George S, Morgan JA, Baldini EH, Wagner AJ, Demetri GD and Raut CP: Leiomyosarcoma of the inferior vena cava: Survival after aggressive management. Ann Surg Oncol 14: 3534-3541, 2007.

6. Dew J, Hansen K, Hammon J, McCoy T, Levine EA and Shen P: Leiomyosarcoma of the inferior vena cava: surgical management and clinical results. Am Surg 71: 497-501, 2005.

7. Kieffer E, Alaoui M, Piette JC, Cacoub P and Chiche L: Leiomyosarcoma of the inferior vena cava: Experience in 22 cases. Ann Surg 244: 289-295, 2006.

8. Mann GN, Mann LV, Levine EA and Shen P: Primary leiomyosarcoma of the inferior vena cava: A 2-institution analysis of outcomes. Surgery 151: 261-267, 2012.
9. Wachtel H, Jackson BM, Bartlett EK, Karakousis GC, Roses RE, Bavaria JE and Fraker DL: Resection of primary leiomyosarcoma of the inferior vena cava (IVC) with reconstruction: A case series and review of the literature. J Surg Oncol 111: 328-333, 2015.

10. Hardwigsen J, Balandraud P, Ananian P, Saïsse J and Le Treut YP: Leiomyosarcoma of the retrohepatic portion of the inferior vena cava: Clinical presentation and surgical management in five patients. J Am Coll Surg 200: 57-63, 2005.

11. Ramponi F, Kench JG, Simring DV, Crawford M, Abadir E and Harris JP: Early diagnosis and resection of an asymptomatic leiomyosarcoma of the inferior vena cava prior to caval obstruction. J Vasc Surg 55: 525-528, 2012.

12. Kulaylat MN, Karakousis CP, Doerr RJ, Karamanoukian HL, O'Brien J and Peer R: Leiomyosarcoma of the inferior vena cava: A clinicopathologic review and report of three cases. J Surg Oncol 65: 205-217, 1997.

13. Trojani M, Contesso G, Coindre JM, Rouesse J, Bui NB, de Mascarel A, Goussot JF, David M, Bonichon F and Lagarde C: Soft-tissue sarcomas of adults; study of pathological prognostic variables and definition of a histopathological grading system. Int J Cancer 33: 37-42, 1984.

14. Gronchi A, Miceli R, Colombo C, Stacchiotti S, Collini P, Mariani L, Sangalli C, Radaelli S, Sanfilippo R, Fiore M and Casali PG: Frontline extended surgery is associated with improved survival in retroperitoneal low- to intermediate-grade soft tissue sarcomas. Ann Oncol 23: 1067-1073, 2012.

15. Toulmonde M, Bonvalot S, Méeus P, Stoeckle E, Riou O, Isambert N, Bompas E, Jafari M, Delcambre-Lair C and Saada E: Retroperitoneal sarcomas: Patterns of care at diagnosis, prognostic factors and focus on main histological subtypes: A multicenter analysis of the French Sarcoma Group. Ann Oncol 25: 735-742, 2014.

16. Kaplan EL and Meier P: Nonparametric estimation from incomplete observations. J Am Stat Assoc 53: 457-481, 1958.

17. Serrano C and George S: Leiomyosarcoma. Hematol Oncol Clin North Am 27: 957-974, 2013.

18. Kwon TW, Sung KB, Cho YP, Kim DK, Yang SM, Ro JY and Kim GE: Pararenal leiomyosarcoma of the inferior vena cava. J Korean Med Sci 18: 355-359, 2003.

19. Perl L: Ein fall von sarkom der vena cava inferior. Arch Path Anat 53: 378-383, 1871

20. Chen E, O'Connell F and Fletcher CD: Dedifferentiated leiomyosarcoma: Clinicopathological analysis of 18 cases. Histopathology 59: 1135-1143, 2011.

21. Cope JS and Hunt CJ: Leiomyosarcoma of inferior vena cava. AMA Arch Surg 68: 752-756, 1954.

22. Kim JT, Kwon T, Cho Y, Shin S, Lee S and Moon D: Multidisciplinary treatment and long-term outcomes in six patients with leiomyosarcoma of the inferior vena cava. J Korean Surg Soc 82: 101-109, 2012.

23. Hines OJ, Nelson S, Quinones-Baldrich WJ and Eilber FR: Leiomyosarcoma of the inferior vena cava: Prognosis and comparison with leiomyosarcoma of other anatomic sites. Cancer 85: 1077-1083, 1999.

24. Mastoraki A, Leotsakos G, Mastoraki S, Papanikolaou IS, Danias N, Smyrniotis V and Arkadopoulos N: Challenging diagnostic and therapeutic modalities for leiomyosarcoma of inferior vena cava. Int J Surg 13: 92-95, 2015.

25. Cacoub P, Piette JC, Wechsler B, Ziza JM, Blétry O, Bahnini A, Kieffer E and Godeau P: Leiomyosarcoma of the inferior vena cava. Experience with 7 patients and literature review. Medicine (Baltimore) 70: 293-306, 1991.

26. Daylami R, Amiri A, Goldsmith B, Troppmann C, Schneider PD and Khatri VP: Inferior vena cava leiomyosarcoma: Is reconstruction necessary after resection? J Am Coll Surg 210: 185-190, 2010.

27. Bower TC, Nagorney DM, Cherry KJ Jr, Toomey BJ, Hallett JW, Panneton JM and Gloviczki P: Replacement of the inferior vena cava for malignancy: An update. J Vasc Surg 31: 270-281, 2000.

28. Kraybill WG, Callery MP, Heiken JP and Flye MW: Radical resection of tumors of the inferior vena cava with vascular reconstruction and kidney autotransplantation. Surgery 121: 31-36, 1997.

29. Hardwigsen J, Baqué P, Crespy B, Moutardier V, Delpero JR and Le Treut YP: Resection of the inferior vena cava for neoplasms with or without prosthetic replacement: A 14-patient series. Ann Surg 233: 242-249, 2001.

30. Sarkar R, Eilber FR, Gelabert HA and Quinones-Baldrich WJ: Prosthetic replacement of the inferior vena cava for malignancy. J Vasc Surg 28: 75-83, 1998. 
31. Illuminati G,Calio' FG, D'Urso A, Giacobbi D,Papaspyropoulos V and Ceccanei G: Prosthetic replacement of the infrahepatic inferior vena cava for leiomyosarcoma. Arch Surg 141: 919-924, 2006.

32. Quinones-Baldrich W, Alktaifi A, Eilber F and Eilber F: Inferior vena cava resection and reconstruction for retroperitoneal tumor excision. J Vasc Surg 55: 1386-1393, 2012.

33. Yamaguchi R, Yamaguchi A, Isogai M, Hori A and Kin $\mathrm{Y}$ : Leiomyosarcoma of the inferior vena cava. Resection and reconstruction of the renal vein using the gonadal vein. Surg Today 28 359-361, 1998.

34. Wang Q, Jiang J, Wang C, Lian G, Jin MS and Cao X: Leiomyosarcoma of the inferior vena cava level II involvement: Curative resection and reconstruction of renal veins. World J Surg Oncol 10: 120, 2012.
35. Angiletta D, Fullone M, Greco L, Marinazzo D, Frontino P and Regina G: Leiomyosarcoma of the inferior vena cava: Resection and vascular reconstruction using a dacron graft and an Adam DeWeese clip-three-year follow-up. Ann Vasc Surg 25: 557 e5-e9, 2011.

36. Arkadopoulos N, Karmaniolou I, Ekonomopoulos N, Vassiliu P and Smyrniotis V: Combination of total abdominal inferior vena cava resection with a novel technique of left renal outflow restoration. Surgery 152: 142-143, 2012. 